\title{
Agricultura urbana e conservação de agrobiodiversidade: um estudo de caso em Mato Grosso, Brasil
}

\author{
Mirella Cultrera ${ }^{1}$, Maria Christina de Mello Amorozo ${ }^{2 *} \&$ Fabio Cop Ferreira ${ }^{3}$
}

${ }^{1}$ Avenida Rio do Peixe, 120, 13950-000, Lindoia, São Paulo, Brasil.

${ }^{2}$ Departamento de Ecologia, IB - UNESP - Caixa Postal 199, 13506-900, Rio Claro, São Paulo, Brasil.

${ }^{3}$ Avenida 20-A, 974, 13506-750, Rio Claro, São Paulo, Brasil.

\begin{abstract}
Resumo - Habitantes de cidades de pequeno porte, muitos deles oriundos de áreas rurais, comumente cultivam plantas alimentares nas zonas urbana e periurbana, podendo contribuir para a conservação da agrobiodiversidade. Este estudo teve o objetivo de investigar esta questão a partir de uma perspectiva etnobotânica. Cinco bairros periféricos da cidade de Santo Antônio do Leverger, em Mato Grosso, foram selecionados. Foi feita uma listagem geral de plantas alimentares cultivadas em 135 unidades domésticas selecionadas aleatoriamente; em 30, foi feito um levantamento etnobotânico pormenorizado. Os dados foram analisados descritivamente e por análises de similaridade e ordenação. Foram identificadas 97 espécies de 38 famílias botânicas, cultivadas em três tipos de áreas: quintais e roças, que apresentam estrutura e composição florística diferentes e complementares; e terrenos vagos, que são intermediários entre ambos. Embora algumas espécies sejam comuns à maioria das unidades domésticas, a similaridade entre os conjuntos de espécies/variedades mantidos é, em geral, menor que 50\%; além disso, boa parte das espécies está em baixa frequência. Isto mostra, por um lado, a contribuição diferenciada de cada unidade doméstica para o acervo total, e por outro, a vulnerabilidade à perda de espécies/variedades, contornada em parte por sua circulação na rede social. Considerando que muitas cidades brasileiras têm as características da área de estudo, o potencial para manutenção de agrobiodiversidade nestas situações deve ser considerado.
\end{abstract}

Palavras-chave adicionais: cidades pequenas, etnobotânica, periferia urbana, plantas alimentares.

\begin{abstract}
Urban agriculture and agrobiodiversity conservation: a case study in Mato Grosso State, Brazil) - It is common for residents of small cities, who are often of rural origin, to grow food plants in urban and peri-urban areas, an activity that can contribute to plant species conservation. An ethnobotanical study was conducted of this phenomenon in five peripheral neighborhoods of the town of Santo Antônio do Leverger, Mato Grosso State, Brazil. A survey of 135 randomlyselected households was carried out to characterize the frequency of food plants, and a more detailed ethnobotanical study was done in 30 households. Descriptive analyses and analyses of similarity and ordination were conducted. Ninetyseven species from 38 botanical families were identified, cultivated in three types of areas: homegardens and fields, which have different and complementary floristic structure and composition; and empty lots, which are intermediary between the other two. While some species are common to the majority of households studied, the similarity between the sets of species/varieties is, in general, below 50\%; besides, many of them have low frequency. On the one hand, this illustrates the different contributions of each household to the total; on the other hand, it also reveals the vulnerability to loss of species/varieties, which is partially avoided through their circulation in the social network. Since many cities in Brazil have characteristics similar to the one studied, their potential for maintaining agro-biodiversity should be taken into consideration.
\end{abstract}

Additional key words: ethnobotany, food plants, small cities, urban periphery.

A agricultura urbana e periurbana ${ }^{1}$, entendida como a realização de atividades de cultivo de plantas e também criação de animais em espaços abertos na malha urbana e periurbana, não é um fenômeno recente (Bryld 2003). Entre outras funções benéficas, como melhorar a qualidade da dieta e a renda de populações carentes, atualmente tem também sido destacado seu potencial para conservação da agrobiodiversidade (Emperaire \& Eloy 2008; Bernholt et al. 2009; Akinnifesi et al. 2010; Thompson et al. 2010; Siviero et al. 2011). Estudos sobre o potencial das periferias urbanas para a manutenção da agrobiodiversidade justificam-se em vista das mudanças que vêm acontecendo nas áreas rurais, com a disseminação do modelo agrícola industrial e a influência do estilo de vida moderno, que colocam em risco

*Autora para correspondência: mcma@rc.unesp.br

Editor responsável: Ulysses Paulino de Albuquerque

Submetido em: 5 out. 2012; publicação eletrônica: $21 \mathrm{dez} .2012$ a manutenção da agrobiodiversidade local tradicionalmente feita pelos agricultores de pequena escala (Bellon 1996; Lamont et al. 1999; Agnihotri \& Palni 2007).

Os migrantes de origem rural que se estabelecem nas periferias das cidades reproduzem aí, até certo ponto, alguns aspectos de seu modo de vida anterior, incluindo o cultivo e a circulação de propágulos (Winklerprins 2002; Emperaire \& Eloy 2008). Evidentemente, a extensão e intensidade destas atividades dependerão das condições e das oportunidades oferecidas. O modo de vida de cidades pequenas, onde há maior disponibilidade de tempo e de espaço, pode favorecer estas práticas, como mostram alguns es-

\footnotetext{
${ }^{1}$ Os dois termos serão usados de forma intercambiável no presente trabalho devido ao tamanho reduzido da cidade e à pouca distância que separa alguns bairros periféricos do centro.
}

Sitientibus série Ciências Biológicas 12(2): 323-332. 2012. 
tudos etnobotânicos em Mato Grosso (Fracaro \& Guarim 2008; Guarim Neto \& Novais 2008; Carniello et al. 2010). Grande parte dos municípios brasileiros (70\%, segundo Veiga 2003) tem pequena área urbanizada, baixa densidade demográfica e boa parte da economia ligada à exploração direta dos recursos naturais, tornando difícil uma separação nítida entre áreas rurais e urbanas. Tais características podem ser favoráveis para a manutenção da agrobiodiversidade na zona urbana e periurbana, merecendo uma investigação mais detalhada.

O município de Santo Antônio do Leverger, em Mato Grosso, possui estas características. Além disso, próximo à sede, encontram-se algumas comunidades de agricultores tradicionais, que foram objeto de estudo anterior (Amorozo 2000). Desta forma, a periferia da cidade de Santo Antônio foi selecionada para investigar se de fato há manutenção de diversidade agrícola e o quanto ela está ligada à zona rural. Assim, os objetivos deste estudo foram: 1- identificar as principais áreas de cultivo utilizadas pelos moradores, descrever sua estrutura e função e verificar se existe um padrão de similaridade entre elas; 2- fazer um levantamento etnobotânico das plantas alimentares cultivadas e identificar as principais formas de obtenção de propágulos; 3- verificar como as unidades domésticas contribuem para a riqueza de espécies cultivadas, quão similares são os conjuntos de plantas mantidos por elas e identificar as espécies mais frequentes.

\section{Material e Métodos}

Área de estudo. O município de Santo Antônio do Leverger (15\%47.11'S, 5604.17'W), Mato Grosso, localiza-se na Baixada Cuiabana, fazendo divisa ao norte com a capital do Estado e ao sul com os Pantanais Matogrossenses. A fisionomia da vegetação na área de estudo é predominantemente de cerrado, embora grande parte dela já esteja modificada pela atividade antrópica. O clima da região tem duas estações bem definidas, um período de chuvas (outubro a março) e um período seco (abril a setembro), sem grandes oscilações de temperatura ao longo do ano, sendo as médias anuais entre $23^{\circ}$ e $25^{\circ} \mathrm{C}$ (Brasil 1982).

O município tem 18.463 habitantes, dos quais um pouco mais de um terço habita a zona urbana (IBGE 2010), que dista cerca de $30 \mathrm{~km}$ da capital do Estado, Cuiabá. As principais atividades econômicas são a agropecuária, pesca artesanal e, mais recentemente, o turismo e a pesca esportiva. As comunidades de agricultores tradicionais próximas à sede do município destacam-se pelo cultivo de mandioca e pela produção de farinha. Mantêm um sistema agrícola diversificado, sobretudo no que diz respeito às etnovariedades de mandioca (cerca de 60) (Amorozo 2000). Nos últimos anos, porém, constataram-se mudanças no uso da terra, pouca continuidade do trabalho agrícola pelas gerações mais jovens, o que ameaça a manutenção deste modo de vida e, por extensão, o acervo de variedades agrícolas até então presente no local (Amorozo 2010). Por outro lado, observou-se expansão da região periférica da sede do município, em função do surgimento de loteamentos particulares e de conjuntos habitacionais.

Foram estudados cinco bairros: Conjunto Habitacional Marechal Rondon (Cohab), Jardim da Laje, Jardim Santo Antônio, Jardim Aeroporto e Fronteira, situados na periferia da cidade e localizados às margens da rodovia MT-040, sentido Santo Antônio do Leverger-Cuiabá. Com exceção do bairro Fronteira, cujo início data do século XIX, os outros bairros foram implantados nas décadas de 1980 e 1990, em áreas de cerrado, que atualmente se encontram bastante alteradas e degradadas; no bairro Fronteira, também há uma faixa de mata ciliar. Todos os bairros contam com sistema de abastecimento de água e eletricidade, mas não coleta de esgotos, e a maior parte das vias públicas não é asfaltada.

Coleta de dados. Foi realizada entre julho de 2006 e abril de 2008. A primeira etapa do trabalho consistiu em fazer um diagnóstico geral sobre a ocorrência das espécies e áreas cultivadas nos bairros selecionados. Após mapeamento esquemático dos bairros, foi feita amostragem aleatória de 135 domicílios $(n=453)$, estratificada a partir da concentração de casas dos bairros ${ }^{2}$ (Tabela 1). Nesta etapa, foi feita uma breve caracterização dos entrevistados e listadas as principais plantas alimentares em seus quintais; não foram visitadas outras áreas de cultivo. Na etapa seguinte, foi utilizada a amostragem por julgamento (Bernard 1988), que teve

Tabela 1. Distribuição das amostras nos bairros estudados na periferia de Santo Antônio do Leverger, Mato Grosso.

\begin{tabular}{lcc}
\hline & Bairros \\
\hline & $\begin{array}{c}\text { Mais populosos } \\
\text { (Fronteira e Cohab) }\end{array}$ & $\begin{array}{c}\text { Menos populosos } \\
\text { Total de domicílios }\end{array}$ \\
$\begin{array}{l}\text { identificados }(\mathrm{n}=453) \\
\text { Domicílios visitados da }\end{array}$ & 324 & 129 \\
$1^{\mathrm{a}}$ amostragem $(\mathrm{n}=135)$ & 76 & 59 \\
$\begin{array}{l}\text { Domicílios visitados da } \\
2^{\mathrm{a}} \text { amostragem }(\mathrm{n}=30)\end{array}$ & 23 & 7 \\
\hline
\end{tabular}

${ }^{2}$ O tamanho amostral foi baseado em Krejcie e Morgan (1970), com nível de significância de 0,1. 
como critério a seleção dos informantes que mantinham o manejo constante de alguma área de cultivo. Foram escolhidas 30 unidades domésticas (famílias habitando domicílios independentes), sendo 21 pertencentes à primeira amostra $\mathrm{e}$ o restante incluído por indicação. Para sua caracterização, foram feitas entrevistas abertas e semiestruturadas (Bernard 1988) com os moradores responsáveis pelo manejo (em 10 domicílios, o casal; em 9, homens; em 11, mulheres). Foram coletados dados socioeconômicos, de história de vida, etnobotânicos e sobre as áreas cultivadas, que foram identificadas pelos próprios moradores. O levantamento etnobotânico das plantas alimentares foi feito com o auxílio da técnica "Turnê Guiada” (Albuquerque \& Lucena 2004), nas áreas de cultivo utilizadas pela unidade doméstica, incluindo todos os quintais e terrenos cultivados, bem como cinco roças (de um total de 12). A circulação dos propágulos de cada espécie cultivada foi registrada; recebimento/doação de propágulos foram conjuntamente denominados "ocorrências". As plantas, em geral espécies bastante conhecidas, foram identificadas em campo; as que não puderam ser identificadas no local (como Ocimum spp. e abóboras), foram coletadas e enviadas a especialistas. O projeto de pesquisa foi aprovado pelo Comitê de Ética em Pesquisa do Instituto de Biociências da UNESP, Campus de Rio Claro, protocolo 6256.

Análise de dados. As análises descritas a seguir foram feitas apenas para os dados da segunda amostragem. As áreas de cultivo foram classificadas como quintais, terrenos e roças, e a riqueza de espécies comparada por curvas de acumulação de espécies (Gotelli \& Colwell 2001). O padrão de similaridade na composição de espécies de quintais, terrenos e roças foi analisado por um escalonamento multidimensional não-métrico (NMDS) utilizando o índice de Jaccard. Para testar a hipótese de que o padrão de similaridade depende do tipo de área, foi aplicada a análise de variância não paramétrica ANOSIM (Legendre \& Legendre 1998). O padrão foi explicado com base no ciclo de vida (anual ou perene) e hábito vegetativo (arbórea, arbustiva, herbácea, herbácea escandente ou liana) das plantas cultivadas.

Os dados provenientes dos quintais permitiram a análise do padrão de similaridade das espécies presentes, avaliada a partir de uma análise de agrupamento utilizando o método de ligação pela média dos grupos e o índice de Jaccard.
Todas as análises foram feitas no pacote estatístico $\mathrm{R}(\mathrm{R}$ Development Core Team 2012).

\section{Resultados}

Caracterização dos entrevistados. Na primeira amostragem, observou-se que quase metade dos habitantes abordados (46\%) nasceu na própria sede do município ou veio de Cuiabá. São oriundos da zona rural de Santo Antônio $26 \%$ dos entrevistados e o restante veio de outros municípios da Baixada Cuiabana e de outros Estados. A metade deles vive, no mínimo, há dez anos na residência atual.

Os moradores mais ativos no cultivo de plantas, selecionados para a segunda etapa do trabalho, são, em sua maioria $(85 \%)$, maiores de 50 anos e provenientes de áreas rurais (75\%). Cerca de $43 \%$ são originários do próprio município, sendo o restante originário de outras localidades em Mato Grosso (32\%) ou de outros Estados (25\%). Cerca de 57\% moram em sua atual residência no mínimo há 10 anos. A maioria tem menos de quatro anos de escolaridade $(62,5 \%)$ ou é analfabeta $(27,5 \%)$. Todos exerceram, em alguma época da vida, atividades agrícolas. A maioria dos homens tem aposentadoria rural $(52 \%)$ e o restante exerce atividades como lavrador/pescador, guarda noturno, funcionário público, caseiro e pedreiro. A maioria das mulheres afirmou ser 'do lar' (67\%). É frequente encontrar nos domicílios mais de uma geração da família ( $50 \%$ com duas gerações; $43,5 \%$ com três gerações), porém, a maior parte dos filhos (70\% do total) já saiu da casa dos pais, estabelecendo residência, em geral, na mesma cidade $(38,5 \%)$ ou na capital do Estado (40,5\%). A escolaridade dos filhos é maior que a dos pais; dos filhos que exercem atividade remunerada $(\mathrm{n}=95)$, apenas $4 \%$ têm alguma atividade ligada à agricultura/cultivo de plantas.

Áreas cultivadas. Foram identificados três principais espaços de cultivo utilizados pelos moradores (Tabela 2): quintais, terrenos vagos nos bairros e roças, nas margens do rio Cuiabá (praia) e em chácaras (campo-fora - terra não alagável). O quintal é o espaço mais utilizado para o cultivo e foi encontrado em quase todas as unidades domésticas de ambas as amostras (97\%). Terrenos e/ou roças, por sua vez, são cultivados por $20 \%$ dos informantes da primeira amostra e por metade dos informantes da segunda amostra: destes últimos, 26,5\% têm uma combinação de quintal e roça,

Tabela 2. Tamanho e situação de posse das áreas de cultivo, Santo Antônio do Leverger, Mato Grosso.

\begin{tabular}{llll}
\hline & Quintal & Terreno & Roça \\
\hline Número de áreas & 29 & 7 & $12(5$ visitadas $)$ \\
Tamanho $\left(\right.$ Média $\left.-\mathrm{m}^{2}\right)$ & 1206,15 & 363,71 & 3400 \\
Amplitude $\left(\mathrm{m}^{2}\right)$ & $207-4339,5$ & $56-720$ & $2000-5000$ \\
Posse da área & Todos próprios & 1 próprio & 2 próprias \\
& & 2 de desconhecidos & 3 de conhecidos \\
\end{tabular}


$16,5 \%$ têm quintal e terreno e os $7 \%$ restantes cultivam as três áreas. A seguir, cada uma delas é descrita brevemente.

Quintais: localizam-se próximo às residências e, além do cultivo de plantas, são utilizados para tarefas domésticas, lazer e convívio social. O manejo geralmente é feito pelas mulheres, que se dedicam às plantas ornamentais, medicinais, protetoras/religiosas e algumas alimentares; os homens se dedicam mais às plantas alimentares que também são cultivadas na roça. Frutíferas e hortaliças são cuidadas por ambos. Onze hortas foram encontradas nos quintais examinados; são áreas isoladas com telas, para proteção contra animais, onde se cultivam hortaliças, plantas medicinais, temperos e mudas de árvores frutíferas, em recipientes, para serem posteriormente transplantadas. Nos quintais estudados, também se cultivam temporariamente, em épocas de cheia do rio Cuiabá, plantas de roça, como mandioca e batata-doce, para que a variedade não seja perdida. Por estar próximo à casa, o quintal também funciona como um local de teste, onde se introduzem sementes recém-obtidas para observar como se comportam antes de cultivá-las na roça.

Terrenos: são áreas urbanas sem edificação, que são aproveitadas para cultivar plantas. Todos são localizados no mesmo bairro em que residem os informantes, próximo às suas moradias. A maioria dos terrenos é manejada por homens. Geralmente, as plantas cultivadas se restringem às alimentares, que também são plantadas em roças. O tempo de cultivo dos terrenos é relativamente curto, em média, de dois anos. Isto evidencia um processo dinâmico e oportunista, relacionado às necessidades familiares (por exemplo, falta de espaço no quintal, manter o terreno limpo para evitar animais indesejáveis) e também à questão da posse dos terrenos, pois a maioria dos informantes não é proprietária da área (Tabela 2). A produção, tanto nos quintais, como nos terrenos, é basicamente para consumo da família, mas há quem doe para amigos e vizinhos.

Roças: entre os informantes da segunda amostra, foram contabilizadas 12 roças: cinco em "chácaras” em áreas de "campo-fora" (cerrado), seis em "praias", localizadas às margens do rio Cuiabá, e uma roça comunitária no bairro Fronteira. As roças visitadas foram cinco, na praia, distantes cerca de dois quilômetros da moradia dos informantes. Geralmente são grandes áreas divididas entre diferentes agricultores que se ajudam mutuamente na época de plantio e colheita. As roças são manejadas, em sua maioria, por homens e, em todas elas, só foram encontradas plantas alimentares, cultivadas após a época das águas, em fevereiro ou março, quando o rio Cuiabá baixa e deixa as terras fertilizadas. Há roças em que se combinam diferentes espécies, por exemplo, feijão-de-corda com milho, melancia, abóbora e milho; mandioca e feijão-de-corda, etc. A produção é, principalmente, para o consumo familiar, venda no comércio local e, no caso do milho, também para ser utilizado como isca na pesca.

O tamanho das três áreas varia dentro de uma faixa de 50 a $5000 \mathrm{~m}^{2}$, sendo as roças, em geral, maiores (Tabela 2).
Do ponto de vista da riqueza de espécies, os quintais são os locais mais ricos, seguidos pelos terrenos e roças. A curva de acumulação mostra que este padrão se mantém, mesmo descontando as diferenças no tamanho amostral (Figura 1; Tabela 2). Percebe-se que, para os quintais e terrenos, as curvas de acumulação de espécies continuam a apresentar um padrão de crescimento, enquanto que, para as áreas de roça, a curva sugere que a riqueza irá se estabilizar em torno de 20 espécies.

Os resultados da ANOSIM evidenciaram efeito significativo $(\mathrm{R}=0,81, \mathrm{p}<0,001)$ do tipo de área de cultivo (quintal, terreno ou roça) sobre o padrão de similaridade. Este padrão sugere dois grandes grupos (Figura 2): o maior, formado pelas áreas de quintais e por três terrenos; e o menor, formado pelas cinco áreas de roça e pelos quatro terrenos restantes. A separação entre eles ocorre ao longo do primeiro eixo do NMDS. Quando caracterizados pelo ciclo de vida das plantas, os padrões mostram um gradiente ao longo do eixo 1 do NMDS, que separa gradualmente quintais, com predomínio de plantas perenes, dos terrenos e roças, com predomínio de plantas anuais (Figura 3A). Considerando os hábitos vegetativos, os quintais são caracterizados ainda por maior variedade de hábitos, enquanto as roças e terrenos do segundo grupo têm predomínio de plantas herbáceas e herbáceas escandentes (Figura 3B). Observa-se também que os terrenos são unidades de cultivo mais heterogêneas, parte deles com características mais próximas aos quintais e parte deles com características mais próximas às roças.

Se considerado o padrão de similaridade somente entre os quintais, não se observam agrupamentos distintos (Figura 4). Os coeficientes de similaridade de Jaccard são, em

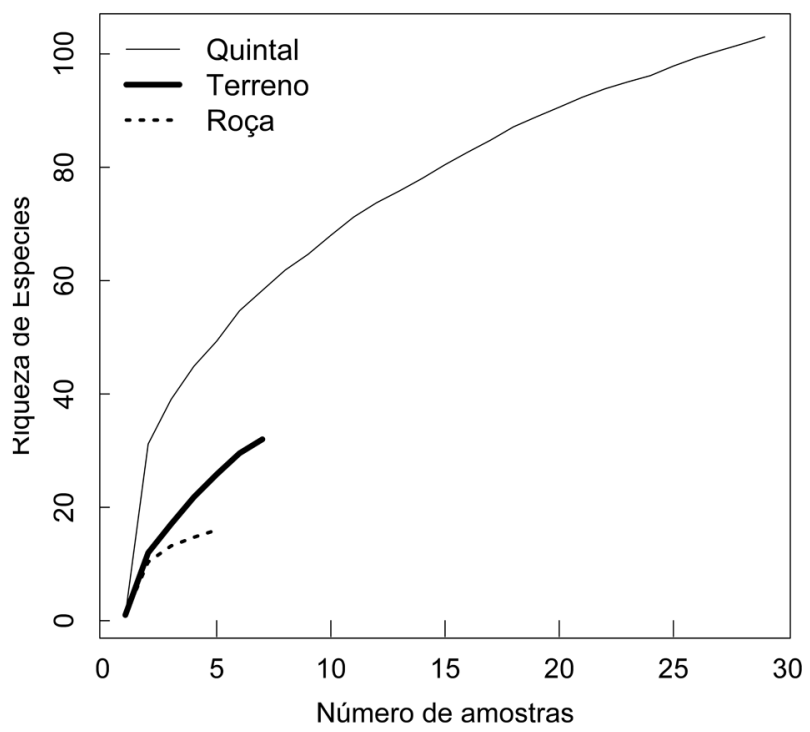

Figura 1. Curva de acumulação de espécies para as três áreas de cultivo (quintais, terrenos e roças). Santo Antônio do Leverger, Mato Grosso, Brasil. 


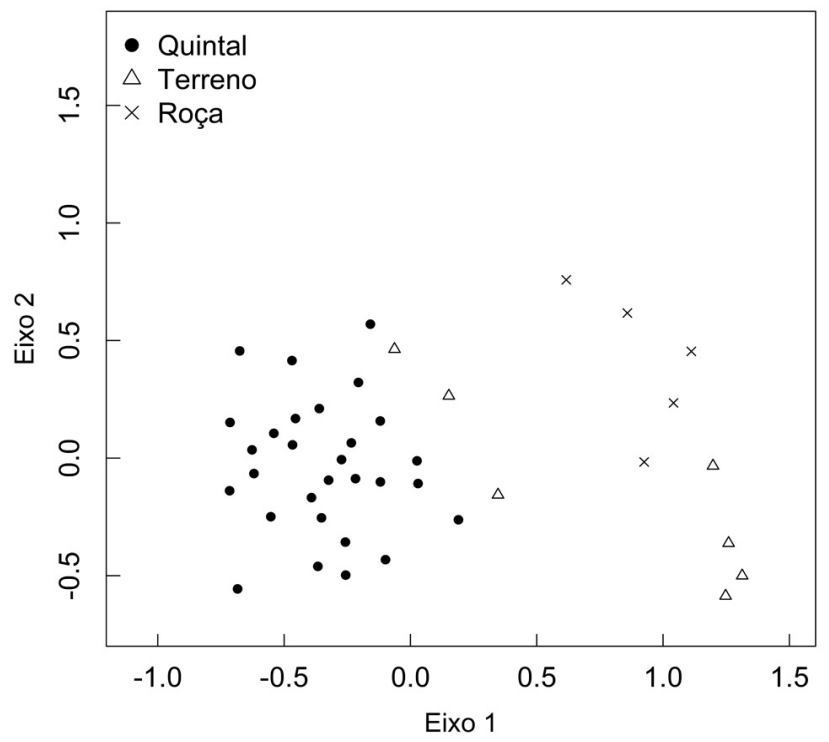

Figura 2. Resultado do escalonamento multidimensional não-métrico (NMDS) para as três áreas de cultivo (quintais, terrenos e roças). Santo Antônio do Leverger, Mato Grosso, Brasil.

sua maioria, baixos, sendo que somente três pares de moradores têm valores acima de 0,5.

Plantas alimentares: dados botânicos e etnobotânicos. Foram registradas 86 espécies na primeira amostragem (apenas quintais) e 97 na segunda (todos os espaços de cultivo) (Tabela 3; Apêndice 1). As famílias botânicas com maior número de espécies, em ambas as amostras, foram Solanaceae (6 espécies na primeira amostra e 7 na segunda), Cucurbitaceae (6 em ambas), Fabaceae (5 e 6),

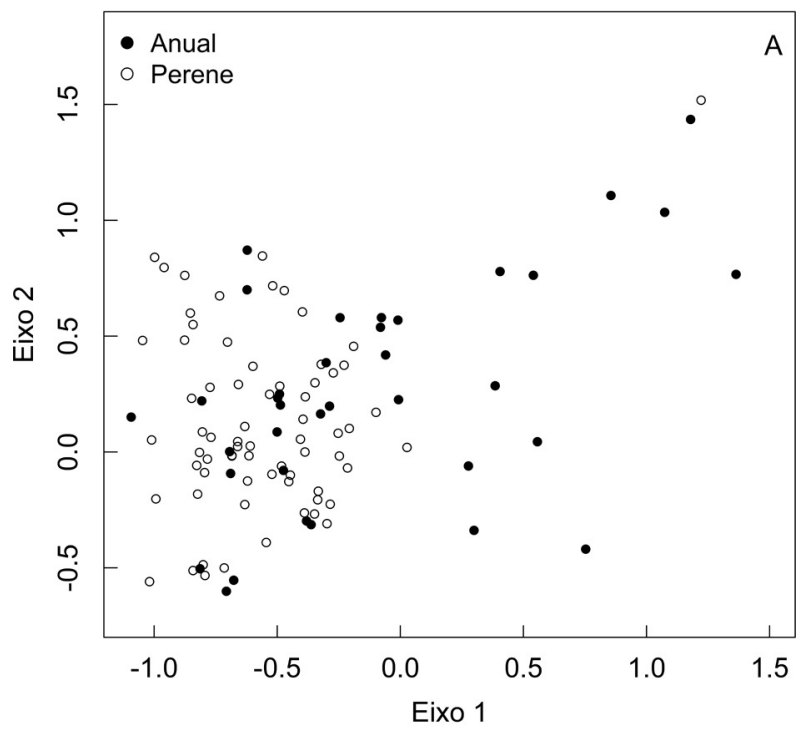

Lamiaceae ( 3 e 6) e Anacardiaceae ( 5 e 4).

Nos quintais, as espécies de frutíferas perenes, como manga, acerola, caju, ata e limões, foram as mais frequentes em ambas as amostras; na segunda amostra, a mandioca e a banana se destacaram, juntamente com as espécies citadas acima. Apenas oito espécies de frutíferas nativas foram encontradas, mantidas nos quintais de bairros mais antigos por informantes que moram no local há mais tempo (Apêndice 1). Moradores de áreas loteadas não tiveram a oportunidade de mantê-las, já que os lotes geralmente são entregues aos proprietários sem a vegetação nativa. Plantas de ciclo curto, como a cebolinha, abóbora e coentro, estão presentes em grande parte dos quintais da segunda amostra, embora, de forma geral, tenham sido menos frequentes que as perenes.

Nas roças, as espécies mais frequentes foram mandioca, milho, abóbora, cana-de-açúcar e maxixe. Nos terrenos, a mandioca, a abóbora e o mamão foram as plantas mais frequentes. Dezessete espécies apresentaram etnovariedades ${ }^{3}$, entre elas, mandioca e banana destacaramse com o maior número: 21 e 14 , respectivamente. As etnovariedades de mandioca mais citadas foram liberata (11) e juruti (10), sendo que esta última é cultivada em todas as roças estudadas, por produzir raízes grandes em apenas seis meses, ideal para ser cultivada às margens do rio Cuiabá, que sofre cheias periódicas. As etnovariedades de banana mais citadas foram banana-da-terra (15) e nanica (9). Das árvores frutíferas, a manga destacou-se, com pelo menos seis variedades, das quais a maioria é antiga e pouco encontrada no comércio atualmente (por exemplo, coquinho, coraçãode-boi, rosa).

Cerca de $52 \%$ das espécies aparecem em $10 \%$ ou me-

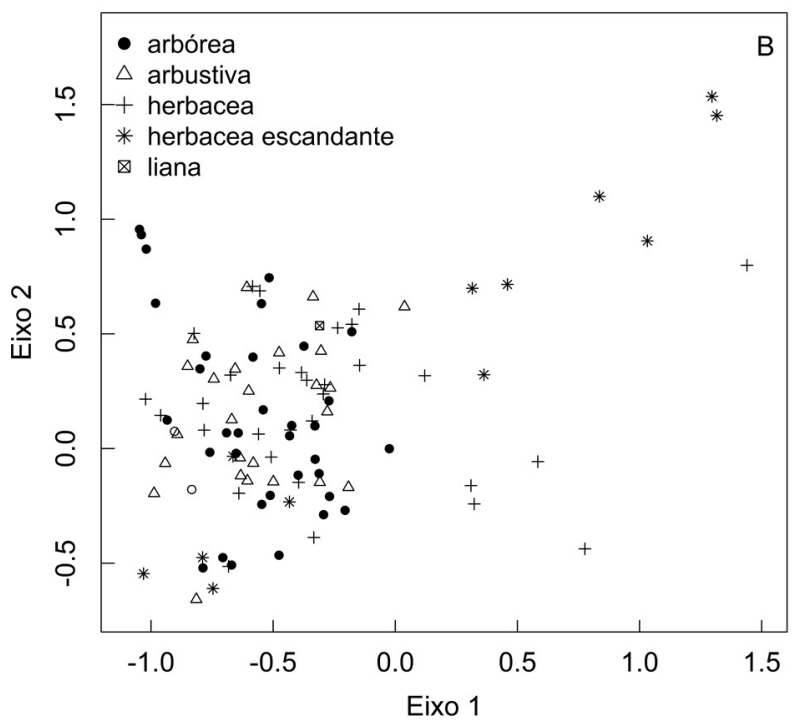

Figura 3. Resultado do escalonamento multidimensional não-métrico (NMDS): A- plantas identificadas segundo seus ciclos de vida (anual ou perene); B- plantas identificadas segundo seus hábitos vegetativos (arbórea, arbustiva, herbácea, herbácea escandente ou liana). Santo Antônio do Leverger, Mato Grosso, Brasil.

${ }^{3}$ Neste estudo, etnovariedades são todas as plantas que foram reconhecidas e identificadas como variedades pelos informantes, não sendo esta classificação necessariamente igual à agronômica. 
Tabela 3. Citações das plantas nos três tipos de áreas de cultivo, Santo Antônio do Leverger, Mato Grosso.

\begin{tabular}{|c|c|c|c|c|c|}
\hline \multirow{2}{*}{ Plantas cultivadas } & \multirow{2}{*}{$\begin{array}{c}1^{\mathrm{a}} \text { Amostragem } \\
\text { Quintal }\end{array}$} & \multicolumn{4}{|c|}{$2^{a}$ Amostragem } \\
\hline & & Total & Quintal & Terreno & Roça \\
\hline Citações/Média & $1084(8)$ & $845(28,1)$ & $736(25,4)$ & $66(9,4)$ & $43(8,6)$ \\
\hline Amplitude de variação & $0-49$ & $13-52$ & $10-52$ & $2-24$ & $5-13$ \\
\hline Número de espécies & 86 & 97 & 89 & 33 & 16 \\
\hline Número de famílias & 35 & 37 & & & \\
\hline
\end{tabular}

nos das unidades domésticas e 38\% são plantas cultivadas (exclusivas) por apenas um morador (Apêndice 1). Avaliando o padrão de similaridade somente entre quintais, não se verificam agrupamentos distintos; o coeficiente de Jaccard entre os pares é, na maioria, menor que 0,5 (Figura 4). Mesmo para os dois moradores com o maior grau de similaridade, o número de plantas em comum fica apenas um pouco acima de $50 \%$ do total.

Obtenção e circulação de material de plantio. O material de plantio utilizado é proveniente dos espaços cultivados pelo próprio agricultor ou obtido a partir de alguma fonte externa, principalmente através da rede social de que faz parte. Esta última foi responsável pela obtenção de cerca de metade das plantas cultivadas nas unidades familiares que tiveram origem externa $(n=696)$. A circulação de propágulos se faz de forma mais intensa na própria zona urbana e periurbana, porém, se estende também à zona rural do município, a outras cidades e mesmo Estados, mas, nos últimos dois casos, são eventos bastante esporádicos. Cerca de $87 \%$ dos entrevistados tinham plantas cujos propágulos vieram da zona rural. A circulação de plantas entre zona urbana e rural no município ( $\mathrm{n}=205$ ocorrências) opera em ambos os sentidos, porém, o fluxo da zona urbana para a rural é menor (cerca de $21 \%$ do total). As plantas com maior circulação de propágulos pela rede social foram mandioca (95 ocorrências), banana (70), acerola (26), cana-de-açúcar (25) e feijões (20). Mandioca e banana foram as que mais circularam entre as zonas urbana e rural.

Todas as unidades domésticas estiveram envolvidas, em maior ou menor proporção, na circulação, com destaque para quatro, que foram as mais ativas. Estas apresentaram algumas características em comum, como número médio maior de espécies cultivadas (39,2 contra 28,1 para toda a amostra) e origem rural dos moradores; três deles têm roças e sempre estiveram envolvidos com atividades agrícolas.

Uma parte das sementes, principalmente de hortaliças, é proveniente do comércio (17\%). A compra de alimentos também é uma forma relativamente comum de obter propágulos (13\%), pois se aproveitam para este fim as sementes dos frutos (como laranja, maracujá, abóbora) ou partes do órgão comestível (como batata-doce e cará).

\section{Discussão}

O cultivo de plantas alimentares é uma prática bastante disseminada na periferia de Santo Antônio do Leverger, sendo os quintais os espaços de cultivo mais frequentes e mais ri- cos. A riqueza média de espécies por quintal $(2,96)$ é semelhante à encontrada em quintais urbanos e periurbanos em outras cidades de pequeno porte (abaixo de 35.000 habitantes) em Mato Grosso. Por exemplo, em Castanheira, foi encontrada uma média de 2,42 espécies alimentares por quintal (Guarim Neto \& Novais 2008); em Juína, 3,2 (Fracaro \& Guarim 2008); e em Mirassol d'Oeste, 3,8 (Carniello et al. 2010).

A análise do NMDS diferenciou claramente os dois principais tipos de áreas de cultivo (quintais e roças), o que é explicado pelo ciclo de vida, hábito vegetativo e riqueza das espécies. Os quintais têm maior riqueza e uma fisionomia vegetacional mais estratificada, abrigando plantas com hábitos vegetativos e ciclos de vida variados, com destaque para as arbóreas perenes, o que é frequentemente relatado na literatura (Coomes \& Ban 2004; Galluzzi et al. 2010). Nas roças, que são áreas mais abertas e mais homogêneas, há predomínio de espécies heliófilas de ciclo curto. Os terrenos são espaços intermediários, ora mais próximos dos quintais, ora das roças, e são plasmados na medida das oportunidades e limitações encontradas. Os conjuntos de espécies presentes nos quintais e roças são diferenciados, embora existam algumas espécies comuns a ambas as áreas, como

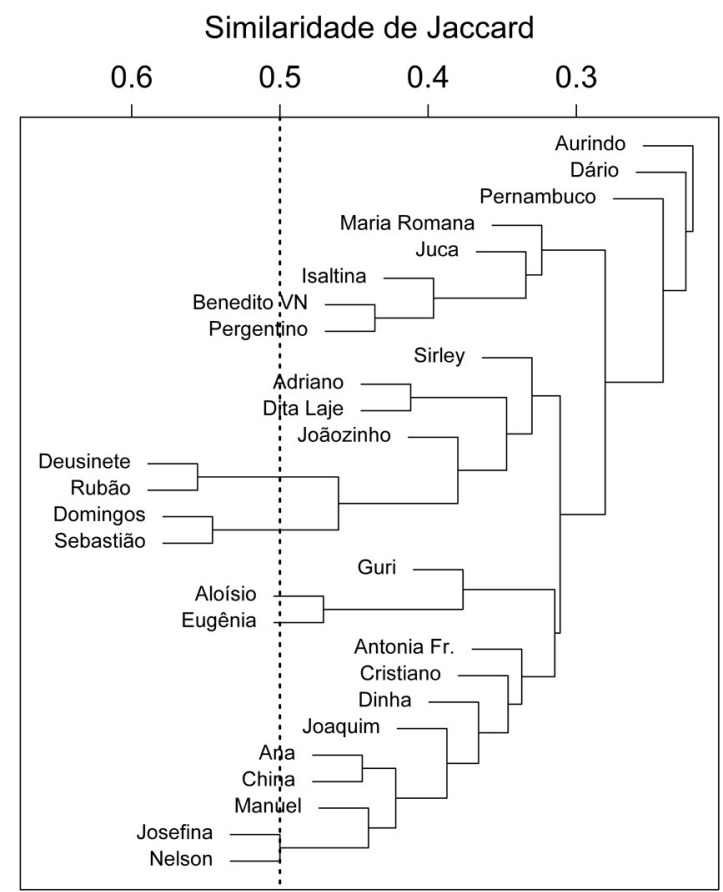

Figura 4. Dendrograma de agrupamento entre moradores nos quintais. Santo Antônio do Leverger, Mato Grosso, Brasil. 
mandioca, batata-doce, bananas e abóboras. Para alguns destes cultivos, o quintal serve como repositório de mudas ou propágulos, até que chegue o tempo de se plantar nas roças. Estes arranjos repetem, até certo ponto, e com maior limitação de área, os encontrados nas comunidades tradicionais próximas, onde quintais e roças também se complementam do ponto de vista estrutural e funcional (Amorozo 1996). Esta articulação e complementaridade proporcionam ao agricultor a oportunidade de manejar uma diversidade maior de plantas alimentares e são aspectos comuns de sistemas agrícolas de pequena escala, como observado por vários autores (e.g., Conelly \& Chaiken 2000; Coomes \& Ban 2004).

Ao examinar a similaridade das unidades domésticas em relação ao conjunto de espécies dos quintais, constata-se mudança gradual de composição ao longo do gráfico, sem a formação de agrupamentos nítidos (Figura 4). Isto contrasta com alguns achados na literatura, por exemplo, o trabalho de Bernholt et al. (2009) em Niger, onde se verificou que os quintais se agrupavam de acordo com a etnia do agricultor e finalidade do cultivo (comercial ou para subsistência), entre outros aspectos. No caso do presente trabalho, a população é relativamente homogênea no que diz respeito a seus hábitos de vida e os produtos dos quintais são basicamente para consumo familiar. Embora exista um pequeno grupo de espécies com grande frequência nas unidades domésticas, a baixa similaridade entre elas permite afirmar que as coleções mantidas pelos agricultores são complementares, dentro do conjunto de espécies observado. Um efeito indesejável desta distribuição seria aumentar o risco de perda a médio e longo prazos para o grupo de espécies "raras" (10\% ou menos de frequência), encontradas apenas em quintais (45 espécies), que são, em geral, espaços de pequenas dimensões e não permitem o cultivo de muitos indivíduos de cada espécie. A circulação de propágulos pela rede social pode diminuir ou evitar estas perdas.

A agricultura urbana aqui abordada está intimamente ligada à área rural. A maior parte das famílias que se destacaram pelas atividades de cultivo na periferia de Santo Antônio é originária da zona rural e teve experiência de vida na agricultura. Vários dos moradores da periferia cultivam suas roças na zona rural e mantêm intercâmbios com as comunidades agrícolas tradicionais. Isto se reflete na composição de seu acervo de espécies e variedades. Por exemplo, no caso da mandioca, os nomes de 10 das etnovariedades plantadas na área periurbana (liberata, juruti, vassourinha, abóbora, uva, pau, matrinchã, cacau, seringueira e mutuana) coincidem com as denominações encontradas nas comunidades tradicionais da zona rural próxima (Amorozo 2000). Embora não tenham sido feitos estudos comparativos, pelo menos algumas delas ( pau, uva, liberata) foram obtidas diretamente nestas comunidades, evidenciando que há circulação de propágulos das variedades e de informação sobre elas entre a zona rural e a urbana abordada neste estudo, o que reforça seu interesse para a conservação da agrobiodiversidade na área. As unidades familiares que têm roças no "campo-fora" ou na "praia" desempenham papel importante, pois, a cada safra, elas atualizam esta articulação, seja através da circulação de propágulos em ambos os sentidos, seja por meio da transferência de cultivos entre roças de beira de rio e os quintais e entre estes e roças de terra firme. Vale lembrar que os membros mais ativos na circulação de propágulos na rede social também plantavam em roças.

A realidade de Santo Antônio do Leverger é semelhante à de grande número de municípios no estado de Mato Grosso e no restante do país, onde ainda se encontram pouca diferenciação entre zona rural e urbana, pequena população e atividades agropecuárias ainda expressivas. A situação de Santo Antônio tem permitido, até agora, que os habitantes da periferia conservem um acervo de espécies e variedades de plantas alimentares em espaços complementares na periferia e na área rural, enriquecido pela sua articulação com as comunidades tradicionais próximas. Mas, até quando será assim? A disponibilidade de terra para plantar roças nos arredores da cidade está diminuindo, com áreas sendo adquiridas para chácaras e residências de fins-de-semana. Emperaire \& Eloy (2008) analisam a recriação da agricultura tradicional indígena com alta agrobiodiversidade na periferia urbana de cidades do Alto e Médio Rio Negro e assinalam também que o acesso mais restrito à terra pode se tornar um problema à sua continuidade. Outra questão a ser considerada no caso de Santo Antônio é que os jovens demonstram pouco interesse pelas atividades de cultivo de seus pais. Apenas uma pequena proporção de descendentes dos moradores entrevistados tinha algum vínculo com a agricultura, $\mathrm{o}$ que leva a incertezas quanto à continuidade destas atividades no futuro. Para o aproveitamento do potencial dos espaços urbanos e periurbanos na manutenção da diversidade agrícola, será necessário esforço sistemático no sentido de lhes dar visibilidade e valorizá-los, procurando despertar o interesse das novas gerações.As políticas públicas atuais de incentivo à pequena agricultura são mais voltadas para segurança alimentar, dando pouca atenção à manutenção e utilização da diversidade agrícola local. É necessário priorizar e valorizar o cultivo de espécies e variedades locais, respeitando os hábitos culturais locais e alcançando a conservação por meio do uso. A maior parte das espécies mais comuns encontradas na área é empregada na culinária regional, como mandioca e banana, consumidas frequentemente em pratos como farofas, ensopados e pirões. Estas são as espécies que apresentam maior número de etnovariedades, o que confirma o fato de que agrobiodiversidade e relevância cultural estão relacionadas. Políticas de valorização da biodiversidade agrícola local deveriam também incluir a proteção e o incentivo ao cultivo de espécies nativas, como pequi (Caryocar 
brasiliense Cambess.), bocaiúva [Acrocomia aculeata (Jacq.) Lodd. ex Mart.] e mangava [Hancornia speciosa Gomes var. pubescens (Nees \& (Mart.) Müll. Arg.], raramente encontradas nos bairros estudados, onde a vegetação do cerrado foi quase toda eliminada. Dado o foco prioritário ao conhecimento local e às perspectivas e valores dos próprios agricultores, trabalhos em Etnobotânica podem, então, contribuir nesta direção.

\section{REFERÊNCIAS}

Agnihotri, R.K. \& Palni, L.M.S. 2007. On-farm conservation of landraces of rice (Oryza sativa L.) through cultivation in the Kumaun Region of Indian Central Himalaya. Journal of Mountain Science 4(4): 354-360.

Akinnifesi, F.K.; Sileshi, G.W.; Ajayi, O.C.; Akinnifesi, A.I.; Moura, E.G.; Linhares, J.F.P. \& Rodrigues, I. 2010. Biodiversity of urban homegardens of São Luís city, Northeastern Brazil. Urban Ecosystems 13: 129-146.

Albuquerque, U.P. \& Lucena, R.F.P. 2004. Métodos e técnicas para coleta de dados. In: U.P. Albuquerque \& R.F.P. Lucena (orgs), Métodos e Técnicas na Pesquisa Etnobotânica. Livro Rápido, Recife, p. 37-63.

Amorozo, M.C.M. 1996. Um Sistema de Agricultura Camponesa em Santo Antônio do Leverger, Mato Grosso, Brasil. Tese de Doutorado, Universidade de São Paulo.

Amorozo, M.C.M. 2000. Management and conservation of Manihot esculenta Crantz. germ plasm by traditional farmers in Santo Antônio do Leverger, Mato Grosso State, Brasil. Etnoecológica 4(6): 68-83.

Amorozo, M.C.M. 2010. Diversidade agrícola em um cenário rural em transformação: será que vai ficar alguém para cuidar da roça? In: L.C. Ming, M.C.M. Amorozo \& C.W. Kffuri (orgs), Agrobiodiversidade no Brasil - experiências e caminhos da pesquisa. NUPEEA, Recife, p. 295-308.

Bellon, M.R. 1996. The dynamics of crop infraspecific diversity: a conceptual framework at the farmer level. Economic Botany 50(1): 26-39.

Bernard, H.R. 1988. Research Methods in Cultural Anthropology. Sage Publications, Newbury Park.

Bernholt, H.; Kehlenbeck, K.; Gebauer, J. \& Buerkert, E. 2009. Plant species richness and diversity in urban and peri-urban gardens of Niamey, Niger. Agroforestry Systems 77: 159-179.

Brasil (Ministério das Minas e Energia) 1982. Folha SD-21 Cuiabá. Levantamento de Recursos Naturais. Projeto RADAMBRASIL. Vol. 26. Secretaria Geral, Rio de Janeiro.

Bryld, E. 2003. Potentials, problems and policy implications for urban agriculture in developing countries. Agriculture and Human Values 20: 79-86.

Carniello, M.A.; Silva, R.S.; Cruz, M.A.B. \& Guarim Neto, G. 2010. Quintais urbanos de Mirassol D’Oeste-MT, Brasil: uma abordagem etnobotânica. Acta Amazonica 40(3): 451-470.

Conelly, W.T. \& Chaiken, M.S. 2000. Intensive farming, agrodiversity and food security under conditions of extreme population pressure in Western Kenya. Human Ecology 28(1): 19-51.

Coomes, O.T. \& Ban, N. 2004. Cultivated plant species diversity in home gardens of an Amazonian peasant village in northeastern Peru. Economic Botany 58(3): 420-434.
Emperaire, L. \& Eloy, L. 2008. A cidade, um foco de diversidade agrícola no Rio Negro (Amazonas, Brasil)? Boletim do Museu Paraense Emilio Goeldi, Ciências Humanas 3(2): 195-211.

Fracaro, F.A. \& Guarim, V.L.M.S. 2008. Uso da biodiversidade em quintais do município de Juína. In: G. Guarim Neto \& M.A. Carniello (orgs), Quintais Matogrossenses - espaços de conservação e reprodução de saberes. UNEMAT Editora, Cáceres, p. 63-78.

Galluzzi, G.; Eyzaguirre, P. \& Negri, V. 2010. Home gardens: neglected hotspots of agro-biodiversity and cultural diversity. Biodiversity Conservation 19: 3635-3654.

Gotelli, N.J. \& Colwell, R.K. 2001. Quantifying biodiversity: procedures and pitfalls in the measurement and comparison of species richness. Ecology Letters 4: 379-391.

Guarim Neto, G. \& Novais, A.M. 2008. Composição florística dos quintais da cidade de Castanheira. In: G. Guarim Neto \& M.A. Carniello (orgs), Quintais Matogrossenses - espaços de conservação e reprodução de saberes. UNEMAT Editora, Cáceres, p. 27-44.

IBGE (Instituto Brasileiro de Geografia e Estatística) 2010. Censo demográfico 2010. Disponível em http://www.ibge.gov.br/ home/estatistica/populaca o/censo $2010 /$ resultados_preliminares; acesso em 22 mar. 2012.

Krejcie, R.V. \& Morgan, D.W. 1970. Determining sample size for research activities. Educational and Psychological Measurement 30: 607-610.

Lamont, S.R; Eshbaugh, W.H. \& Greenberg, A.M. 1999. Species composition, diversity and use of homegardens among three Amazonian villages. Economic Botany 53(3): 312-326.

Legendre, P. \& Legendre, L. 1998. Numerical Ecology. Elsevier Science, Amsterdam.

R Development Core Team 2012. R: a language and environment for statistical computing. R Foundation for Statistical Computing, Vienna. Disponível em http://www.R-project.org/

Siviero, A.; Delunardo, T.A.; Haverroth, M.; Oliveira, L.C. \& Mendonça, A.M.S. 2011. Cultivo de espécies alimentares em quintais urbanos de Rio Branco, Acre, Brasil. Acta Botanica Brasilica 25(3): 549-556.

Thompson, J.L.; Gebauer, J.; Hammer, K. \& Buerkert, A. 2010. The structure of urban and peri-urban gardens in Khartoum, Sudan. Genetic Resources and Crop Evolution 57: 487-500.

Veiga, J. E. 2003. Cidades Imaginárias - o Brasil é menos urbano do que se calcula. 2 ed. Editora Autores Associados, Campinas.

Winklerprins, A.M.G.A. 2002. House-lot gardens in Santarém, Pará, Brazil: linking rural with urban. Urban Ecosystems 6: 43-65. 
Apêndice 1. Plantas alimentares cultivadas por moradores da periferia de Santo Antônio do Leverger, Mato Grosso. Local de cultivo: $\mathrm{Q}=\mathrm{quintal} ; \mathrm{T}=$ terreno; $\mathrm{R}=$ roça. Hábito: $\mathrm{A}=$ arbórea; $\mathrm{AB}=$ arbustiva; $\mathrm{H}=$ herbácea; $\mathrm{HE}=$ herbácea escandente; $\mathrm{L}=$ liana. Ciclo da Planta: $\mathrm{A}=$ anual; $\mathrm{P}=$ perene. $\mathrm{N} . \mathrm{I}$ $=$ não identificado. Nome local em negrito $=$ etnovariedades. $(*)=$ nativas do cerrado.

\begin{tabular}{|c|c|c|c|c|c|c|}
\hline \multirow[t]{2}{*}{ Família/ Nome Científico } & \multirow[t]{2}{*}{ Nome local } & \multicolumn{2}{|c|}{$\begin{array}{l}\text { Frequência nos } \\
\text { domicílios (\%) }\end{array}$} & \multirow[t]{2}{*}{ Local } & \multirow[t]{2}{*}{ Hábito } & \multirow[t]{2}{*}{$\begin{array}{l}\text { Ciclo da } \\
\text { planta }\end{array}$} \\
\hline & & $\begin{array}{c}1^{\mathrm{a}} \\
\text { amostra } \\
\mathrm{n}=135\end{array}$ & $\begin{array}{c}\mathbf{2}^{\mathbf{a}} \\
\mathbf{n}=\mathbf{a m o s}\end{array}$ & & & \\
\hline $\begin{array}{l}\text { ANACARDIACEAE } \\
\text { Anacardium occidentale } \mathrm{L} \text {. } \\
\text { Mangifera indica } \mathrm{L} \text {. }\end{array}$ & $\begin{array}{l}\text { Caju, caju-a marelo, caju-vermelho, caju-anão, caju-castanha-grande } \\
\text { Manga, manga-borbom, adem, coco-de-garrote, coquinho, coração-de- } \\
\text { boi, espada, janeira, janeirinha, ouro, ovo, pequi, porvilho, bola, rosa, } \\
\text { manguinha-peito-de-moça. }\end{array}$ & $\begin{array}{l}43 \\
63,7\end{array}$ & $\begin{array}{l}70 \\
83,3\end{array}$ & $\begin{array}{l}\mathrm{Q} \\
\mathrm{Q} / \mathrm{T}\end{array}$ & $\begin{array}{l}\text { A } \\
\text { A }\end{array}$ & $\begin{array}{l}\mathrm{P} \\
\mathrm{P}\end{array}$ \\
\hline Spondias sp. 1 & Cajá & 37 & - & Q & A & $\mathrm{P}$ \\
\hline Spondias sp. 2 & Cajá-manga & 6,6 & 10 & Q & A & $\mathrm{P}$ \\
\hline Spondias sp. 3 & Ciriguela, jacote & 4,4 & 16,6 & Q & A & $\mathrm{P}$ \\
\hline $\begin{array}{l}\text { ANNONACEAE } \\
\text { Annona squamosa } \mathrm{L} \text {. } \\
\text { Annona muricata } \mathrm{L} \text {. }\end{array}$ & $\begin{array}{l}\text { Ata, fruta-do-conde } \\
\text { Graviola }\end{array}$ & $\begin{array}{l}42,2 \\
3,7\end{array}$ & $\begin{array}{l}80 \\
6,6\end{array}$ & $\begin{array}{l}\mathrm{Q} / \mathrm{T} \\
\mathrm{Q}\end{array}$ & $\begin{array}{l}\text { A } \\
\text { A }\end{array}$ & $\begin{array}{l}\mathrm{P} \\
\mathrm{P}\end{array}$ \\
\hline $\begin{array}{l}\text { APIACEAE } \\
\text { Coriandrum sativum } \mathrm{L} \text {. } \\
\text { Daucus carota } \mathrm{L} \text {. } \\
\text { Eryngium foetidum } \mathrm{L} \text {. } \\
\text { Petroselinum crispum } \\
\quad \text { (Mill.) A.W.Hill } \\
\text { APOCYNACEAE }\end{array}$ & $\begin{array}{l}\text { Coentro } \\
\text { Cenoura } \\
\text { Coentro-da-Índia, castelo } \\
\text { Salsa }\end{array}$ & $\begin{array}{l}11,8 \\
- \\
2,2 \\
1,5\end{array}$ & $\begin{array}{l}43,3 \\
3,3 \\
13,3 \\
16,6\end{array}$ & $\begin{array}{l}\mathrm{Q} \\
\mathrm{Q} \\
\mathrm{Q} \\
\mathrm{Q}\end{array}$ & $\begin{array}{l}\mathrm{H} \\
\mathrm{H} \\
\mathrm{H} \\
\mathrm{H}\end{array}$ & $\begin{array}{l}\text { A } \\
\text { A } \\
\text { A } \\
\text { A }\end{array}$ \\
\hline $\begin{array}{l}\text { Hancornia speciosa Gomes } \\
\text { var. pubescens (Nees \& } \\
\text { Mart.) Müll. Arg. }(*)\end{array}$ & Mangava & - & 3,3 & Q & A & $\mathrm{P}$ \\
\hline ARACEAE & & & & & & \\
\hline Colocasia sp. & Inhame-rosa & - & 3,3 & $\mathrm{Q}$ & $\mathrm{H}$ & A \\
\hline Xanthosoma sp. 1 & Taioba & 0,74 & 3,3 & $\mathrm{Q}$ & $\mathrm{H}$ & A \\
\hline Xanthosoma sp. 2 & Inhame & 0,74 & 3,3 & Q & $\mathrm{H}$ & A \\
\hline ARECACEAE & & & & & & \\
\hline $\begin{array}{l}\text { Acrocomia aculeata (Jacq.) } \\
\text { Lodd. ex Mart. }\left({ }^{*}\right)\end{array}$ & Bocaiúva & 12,6 & 16,6 & $\mathrm{Q} / \mathrm{T}$ & A & $P$ \\
\hline Cocos nucifera $\mathrm{L}$. & Coco, coco-da-Bahia, coco-anão, coco-amarelo & 35,5 & 76,6 & $\mathrm{Q} / \mathrm{T}$ & A & $P$ \\
\hline $\begin{array}{l}\text { Orbignya phalerata Mart. } \\
\left({ }^{*}\right)\end{array}$ & Babaçu & 1,5 & 13,3 & Q & A & $P$ \\
\hline $\begin{array}{l}\text { Syagrus oleraceae (Mart.) } \\
\text { Becc. }\left(^{\star}\right)\end{array}$ & Palmito-amargo & - & 3,3 & Q & A & $P$ \\
\hline $\begin{array}{l}\text { ASTERACEAE } \\
\text { Chicorium } \mathrm{sp}\end{array}$ & & & & & & \\
\hline Chicorium $\mathrm{sp}$ & Almeirão & 1,5 & 3,3 & Q & $\mathrm{H}$ & A \\
\hline Lactuca sativa $\mathrm{L}$. & Alface & 4,4 & 13,3 & $\mathrm{Q}$ & $\mathrm{H}$ & A \\
\hline BRASSICACEAE & & & & & & \\
\hline Brassica oleracea L. & Couve, repolho, couve-mostarda & 3,7 & 29,9 & $\mathrm{Q} / \mathrm{T}$ & $\mathrm{H}$ & A \\
\hline Eruca sativa Mill. & Rúcula & 1,5 & 13,3 & $\mathrm{Q}$ & $\mathrm{H}$ & A \\
\hline Nasturtium officinale $\mathrm{R} . \mathrm{Br}$. & Agrião & 0,74 & - & $\mathrm{Q}$ & $\mathrm{H}$ & A \\
\hline $\begin{array}{l}\text { BIXACEAE } \\
\text { Bixa orellana } \mathrm{L} \text {. }\end{array}$ & Colorau, urucum & - & 10 & $\mathrm{Q}$ & A & $\mathrm{P}$ \\
\hline $\begin{array}{l}\text { BROMELIACEAE } \\
\text { Ananas comosus (L.) Merril. }\end{array}$ & Abacaxi, abacaxi-pérola & 9,6 & 40 & Q & $\mathrm{H}$ & A \\
\hline $\begin{array}{l}\text { CACTACEAE } \\
\text { Pereskia grandifolia } \mathrm{L} .\end{array}$ & Oropronobis & 0,74 & 3,3 & Q & A & $P$ \\
\hline $\begin{array}{l}\text { CARICACEAE } \\
\text { Carica papaya } \mathrm{L} \text {. }\end{array}$ & $\begin{array}{l}\text { Mamão, mamão-castelo, mamão-goiaba, mamão-papaya, mamão- } \\
\text { caseiro, mamão-macho }\end{array}$ & 33,3 & 73,3 & $\mathrm{Q} / \mathrm{T}$ & A & $P$ \\
\hline $\begin{array}{l}\text { CARYOCARACEAE } \\
\text { Caryocar brasiliense } \\
\text { Cambess. }(*)\end{array}$ & Pequi & 8,9 & 13,3 & Q & A & $P$ \\
\hline $\begin{array}{l}\text { CONVOLVULACEAE } \\
\text { Ipomoea batatas }(\mathrm{L} .) \text { Lam. }\end{array}$ & $\begin{array}{l}\text { Batata-doce, batata-doce-amarela, batata-doce-branca, batata-doce- } \\
\text { de-arroba, batata-doce-maxuxo-amarela, batata-doce-maxuxo-branca, } \\
\text { batata-doce-roxa }\end{array}$ & 8,1 & 36,6 & $\mathrm{Q} / \mathrm{T} / \mathrm{R}$ & $\mathrm{HE}$ & A \\
\hline $\begin{array}{l}\text { CUCURBITACEAE } \\
\text { Citrullus lanatus (Thunb.) } \\
\text { Matsum. \& Nakai }\end{array}$ & ( & 5,9 & 10 & $\mathrm{Q} / \mathrm{T} / \mathrm{R}$ & $\mathrm{HE}$ & A \\
\hline Cucumis anguria $\mathrm{L}$. & Maxixe & 2,9 & 16,6 & $\mathrm{Q} / \mathrm{T} / \mathrm{R}$ & $\mathrm{HE}$ & A \\
\hline Cucumis melo $\mathrm{L}$. & Melão & 1,5 & 10 & $\mathrm{Q} / \mathrm{R}$ & $\mathrm{HE}$ & A \\
\hline Cucumis sativus $\mathrm{L}$. & Pepino & 1,5 & 3,3 & $\mathrm{R}$ & $\mathrm{HE}$ & A \\
\hline $\begin{array}{l}\text { Cucurbita spp. } \\
\text { Sechium edule (Jacq.)Sw. }\end{array}$ & $\begin{array}{l}\text { Abóbora, cabotiá } \\
\text { Chuchu }\end{array}$ & $\begin{array}{l}9,6 \\
1,5\end{array}$ & $\begin{array}{l}53,3 \\
3,3\end{array}$ & $\begin{array}{l}\mathrm{Q} / \mathrm{T} / \mathrm{R} \\
\mathrm{Q}\end{array}$ & $\begin{array}{l}\mathrm{HE} \\
\mathrm{HE}\end{array}$ & $\begin{array}{l}\mathrm{A} \\
\mathrm{P}\end{array}$ \\
\hline $\begin{array}{l}\text { Sechium edule (Jacq.)Sw. } \\
\text { DIOSCOREACEAE }\end{array}$ & Cruchu & & & & & \\
\hline Dioscorea alata $\mathbf{L}$. & Cará, cará-roxo & 0,74 & 16,6 & $\mathrm{Q} / \mathrm{T}$ & $\mathrm{HE}$ & A \\
\hline Dioscorea bulbifera $\mathrm{L}$. & Cará & 0,74 & 3,3 & $\mathrm{Q}$ & $\mathrm{HE}$ & A \\
\hline EBENACEAE & & & & & & \\
\hline Diospyros kaki L. f. & Caqui & 2,2 & 10 & Q & A & $\mathrm{P}$ \\
\hline EUPHORBIACEAE & & & & & & \\
\hline Manihot esculenta Crantz & $\begin{array}{l}\text { Mandioca, aipim, impim, mandioca-amarela, vermelha, branca, roxa, } \\
\text { da-rama-preta, mandioca-goiaba, cenoura, uva, abóbora, cacau, alecrim, } \\
\text { comigo-ninguém-pode, folhagem, colorida, seringueira, pau, manteiga, } \\
\text { vassourinha, matrinchã, mutuana, três-meses, seis-meses, mandioca- } \\
\text { juruti, liberata, liberatona, liberata-folha-larga, liberata-folha-fina, } \\
\text { paraguaia ou para-churrasco, arizona, do-Paraná }\end{array}$ & 30,3 & 100 & $\mathrm{Q} / \mathrm{T} / \mathrm{R}$ & $\mathrm{H}$ & A \\
\hline FABACEAE & & & & & & \\
\hline Arachis hypogaea $\mathrm{L}$. & Amendoim-cavalo, amendoim-vermelho & 0,74 & 6,6 & Q & $\mathrm{H}$ & A \\
\hline Hymenaea sp* & Jatobá & 0,74 & - & Q & A & $\mathrm{P}$ \\
\hline Inga sp. & Ingá & 2,9 & 6,6 & Q & A & $\mathrm{P}$ \\
\hline Inga edulis Mart. & Ingá-de-metro & - & 6,6 & Q & A & $\mathrm{P}$ \\
\hline Phaseolus vulgaris $\mathrm{L}$. & Feijão-carioca, carioquinha & - & 3,3 & $\mathrm{R}$ & $\mathrm{HE}$ & A \\
\hline Tamarindus indica $\mathrm{L}$. & Tamarindo, tamarino & 5,2 & 20 & Q & A & $\mathrm{P}$ \\
\hline $\begin{array}{l}\text { Vigna unguiculata }(\mathrm{L} .) \\
\text { Walp. }\end{array}$ & Feijão-trepador, de vara, chicote, de corda, catador, de metro & 5,9 & 20 & $\mathrm{Q} / \mathrm{T} / \mathrm{R}$ & $\mathrm{HE}$ & A \\
\hline N.I. & Feijão-ervilha & - & 3,3 & Q & $\mathrm{HE}$ & A \\
\hline N.I. & Feijão-miúdo & - & 3,3 & Q & $\mathrm{HE}$ & A \\
\hline N.I. & Feijão-vagem-de-salada & - & 3,3 & Q & $\mathrm{HE}$ & A \\
\hline
\end{tabular}




\begin{tabular}{|c|c|c|c|c|c|c|}
\hline \multicolumn{7}{|l|}{ LAMIACEAE } \\
\hline Mentha spp. & Hortelã & 3,7 & 6,6 & $\mathrm{Q}$ & $\mathrm{H}$ & A \\
\hline Ocimum gratissimum $\mathrm{L}$. & Alfavaca & - & 6,6 & Q & $\mathrm{AB}$ & $\mathrm{P}$ \\
\hline Ocimum selloi Benth. & Alfavaquinha & - & 3,3 & $\mathrm{Q}$ & $\mathrm{AB}$ & $\mathrm{P}$ \\
\hline Ocimum sp.1 & Alfavacona & - & 3,3 & $\mathrm{Q}$ & $\mathrm{AB}$ & $\mathrm{P}$ \\
\hline $\begin{array}{l}\text { Plectranthus amboinicus } \\
\text { (Lour.) Spreng. }\end{array}$ & Hortelã-grosso, hortelazão & 0,74 & 6,6 & $\mathrm{Q}$ & $\mathrm{H}$ & A \\
\hline Vitex cymosa Bertero ex & Tarumã & 0,74 & 3,3 & Q & A & $\mathrm{P}$ \\
\hline \multicolumn{7}{|l|}{ Spreng $(*)$} \\
\hline N.I. & Manjericão & 0,74 & - & $\mathrm{Q}$ & $\mathrm{AB}$ & $\mathrm{P}$ \\
\hline N.I. & Manjerona & 1,5 & - & Q & $\mathrm{AB}$ & $\mathrm{P}$ \\
\hline \multicolumn{7}{|l|}{ LAURACEAE } \\
\hline $\begin{array}{l}\text { Cinnamomum zeylanicum } \\
\text { Breyn. }\end{array}$ & Canela & - & 3,3 & Q & A & $\mathrm{P}$ \\
\hline Persea americana Mill. & Abacate & 11,8 & 46,6 & Q & A & $\mathrm{P}$ \\
\hline \multicolumn{7}{|l|}{ LILIACEAE } \\
\hline Allium fistulosum $\mathrm{L}$. & Cebolinha & 28,8 & 70 & $\mathrm{Q} / \mathrm{T} / \mathrm{R}$ & $\mathrm{H}$ & A \\
\hline Allium sativum $\mathrm{L}$. & Alho & - & 10 & & $\mathrm{H}$ & A \\
\hline Allium spp. & Alho, alho-poro, caseiro, de-folha, japonês & 2,2 & 10 & $\mathrm{Q}$ & $\mathrm{H}$ & A \\
\hline LYTHRACEAE & & & & & & \\
\hline Punica granatum $\mathrm{L}$. & Romã & 5,9 & 30 & $\mathrm{Q} / \mathrm{T}$ & $\mathrm{AB}$ & $\mathrm{P}$ \\
\hline MALPIGHIACEAE & & & & & & \\
\hline $\begin{array}{l}\text { Malpighia spp. } \\
\text { Bunchosia sp. }\end{array}$ & $\begin{array}{l}\text { Acerola } \\
\text { Cereja }\end{array}$ & $\begin{array}{l}59,2 \\
0,74\end{array}$ & $\begin{array}{l}80 \\
3,3\end{array}$ & $\begin{array}{l}\mathrm{Q} / \mathrm{T} \\
\mathrm{Q}\end{array}$ & $\begin{array}{l}\mathrm{AB} \\
\mathrm{AB}\end{array}$ & $\begin{array}{l}\mathrm{P} \\
\mathrm{P}\end{array}$ \\
\hline MALVACEAE & & & & & & \\
\hline Abelmoschus esculentus $\mathrm{L}$. & Quiabo & 5,9 & 40 & $\mathrm{Q} / \mathrm{T} / \mathrm{R}$ & $\mathrm{H}$ & A \\
\hline MORACEAE & & & & & & \\
\hline $\begin{array}{l}\text { Artocarpus heterophyllus } \\
\text { Lam. }\end{array}$ & Jaca & 4,4 & 23,3 & Q & A & $P$ \\
\hline Ficus carica $\mathrm{L}$. & Figo & 2,2 & 16,6 & $\mathrm{Q} / \mathrm{T}$ & $\mathrm{AB}$ & $\mathrm{P}$ \\
\hline Morus nigra $\mathrm{L}$. & Amora & 3,7 & 6,6 & $\mathrm{Q}$ & A & $\mathrm{P}$ \\
\hline MUSACEAE & & & & & & \\
\hline Musa spp. & $\begin{array}{l}\text { Banana, bananinha, banana-de-fritar, banana-da-terra, banana-d, } \\
\text { água, três-quinas, roxa, prata, ouro, maçã, nanica, nanicão, missu, } \\
\text { sartaveaco, maricota, mariquita }\end{array}$ & 33,3 & 90 & $\mathrm{Q} / \mathrm{T} / \mathrm{R}$ & $\mathrm{H}$ & $\mathrm{P}$ \\
\hline MYRTACEAE & & & & & & \\
\hline Eugenia uniflora $\mathrm{L}$. & Pitanga & 2,9 & 16,6 & $\mathrm{Q} / \mathrm{T}$ & $\mathrm{AB}$ & $\mathrm{P}$ \\
\hline Myrciaria spp. & Jabuticaba, jabuticava & 5,9 & 20 & $\mathrm{Q} / \mathrm{T}$ & A & $\mathrm{P}$ \\
\hline Psidium guajava $\mathrm{L}$. & Goiaba, goiaba-vermelha, goiaba-branca & 32,6 & 50 & $\mathrm{Q} / \mathrm{T}$ & A & $\mathrm{P}$ \\
\hline Psidium sp. & Goiabinha-do-mato & - & 3,3 & $\mathrm{Q}$ & & \\
\hline Syzygium cumini (L.) Skeels & João-bulão & 0,74 & 3,3 & $\mathrm{Q}$ & A & $\mathrm{P}$ \\
\hline Syzygium jambos (L.) Alston & Jambo, jambo-amarelo & - & 13,3 & $\mathrm{Q}$ & A & $\mathrm{P}$ \\
\hline Syzygium malaccense (L.) & Jambo-roxo & - & 3,3 & Q & A & $\mathrm{P}$ \\
\hline Merr.\& L.M. Perry & & & & & & \\
\hline N.I. & Araçá & 0,74 & - & $\mathrm{Q}$ & A & $\mathrm{P}$ \\
\hline N.I. & Gariroba & 1,5 & 3,3 & $\mathrm{Q}$ & A & $\mathrm{P}$ \\
\hline $\begin{array}{l}\text { OXALIDACEAE } \\
\text { Averrhoa carambola } \mathrm{L} \text {. }\end{array}$ & Carambola & 5,9 & 26,6 & $\mathrm{Q}$ & $\mathrm{AB}$ & $\mathrm{P}$ \\
\hline PASSIFLORACEAE & Caldinvora & & & & & \\
\hline Passiflora edulis Sims & Maracujá, maracujá-azedo, maracujá-pequeno, maracujina. & 5,9 & 60 & $\mathrm{Q}$ & $\mathrm{HE}$ & $\mathrm{P}$ \\
\hline $\begin{array}{l}\text { Passiflora quadrangularis } \\
\text { L. }\end{array}$ & Maracujá-grande, maracujá-castelo & - & 6,6 & $\mathrm{Q}$ & $\mathrm{HE}$ & $\mathrm{P}$ \\
\hline Passiflora spp. & Maracujá, maracujá-do-mato, doce, maracujina & 11,1 & - & $\mathrm{Q}$ & $\mathrm{HE}$ & $\mathrm{P}$ \\
\hline POACEAE & & & & & & \\
\hline Saccharum officinarum $\mathrm{L}$. & $\begin{array}{l}\text { Cana, cana-caiana, caiana-listrada, cristalina, cristalina-listrada, } \\
\text { paranaense, paulista, taquara ou muquinha, para-ração }\end{array}$ & 10,4 & 50 & $\mathrm{Q} / \mathrm{R}$ & $\mathrm{H}$ & A \\
\hline Zea mays $L$. & Milho, milho-verde, 1051, catetinho, comprado & 2,2 & 16,6 & $\mathrm{Q} / \mathrm{R}$ & $\mathrm{H}$ & A \\
\hline ROSACEAE & & & & & & \\
\hline $\begin{array}{l}\text { Fragaria } \times \text { ananassa } \\
\text { (Weston) Duchesne ex } \\
\quad \text { Rozier }\end{array}$ & Morango & - & 10 & $\mathrm{Q}$ & $\mathrm{H}$ & $\mathrm{P}$ \\
\hline Malus $\mathrm{x}$ domestica Borkh. & Maçã & 0,74 & 10 & $\mathrm{~T}$ & $\mathrm{AB}$ & $\mathrm{P}$ \\
\hline Pyrus communis $\mathrm{L}$. & Pêra & 2,2 & 3,3 & $\mathrm{~T}$ & $\mathrm{AB}$ & $\mathrm{P}$ \\
\hline RUBIACEAE & & & & & & \\
\hline Coffea arabica $\mathrm{L}$. & Café & 0,74 & 6,6 & Q & $\mathrm{AB}$ & $\mathrm{P}$ \\
\hline Cordiera sp. $(*)$ & Marmelada-preta & 0,74 & 3,3 & $\mathrm{Q}$ & $\mathrm{AB}$ & $\mathrm{P}$ \\
\hline Genipa americana $\mathrm{L}$. & Jenipapo & 0,74 & 3,3 & $\mathrm{Q}$ & A & $\mathrm{P}$ \\
\hline RUTACEAE & & & & & & \\
\hline Citrus spp. & $\begin{array}{l}\text { Laranja, comum, azeda, de-enxerto, pêra, pêra-São-Paulo, baiana, } \\
\text { laranja-d'água, da-terra, de-fazer-doce, misteriosa. }\end{array}$ & 35,5 & 70 & $\mathrm{Q} / \mathrm{T}$ & A & $\mathrm{P}$ \\
\hline Citrus spp. & Laranja-lima, lima-de-imbigo, lisa, das-peça, da-pérsia & 6,6 & 40 & $\mathrm{Q} / \mathrm{T}$ & A & $\mathrm{P}$ \\
\hline Citrus spp. & $\begin{array}{l}\text { Limão, limão-taiti, galeguinho, galego, limão-cidra, limão-nosso, } \\
\text { caipira, rosa, vermelho. }\end{array}$ & 41,5 & 80 & $\mathrm{Q} / \mathrm{T}$ & A & $\mathrm{P}$ \\
\hline Citrus spp. & Ponkan, laranja-ponkan, morgote, tangerina, mexerica & 14,8 & 30 & $\mathrm{Q} / \mathrm{T}$ & A & $\mathrm{P}$ \\
\hline SAPINDACEAE & & & & & & $\mathrm{P}$ \\
\hline $\begin{array}{l}\text { Talisia esculenta (A.St.- } \\
\text { Hil.) Radlk. }\end{array}$ & Pitomba & 5,2 & 16,6 & Q & A & $\mathrm{P}$ \\
\hline SOLANACEAE & & & & & & \\
\hline Capsicum annuum $\mathrm{L}$. & Pimentão & 8,9 & 26,6 & $\mathrm{Q} / \mathrm{T}$ & $\mathrm{H}$ & A \\
\hline Capsicum chinense Jacq. & Pimenta-de-cheiro, pimenta-de-cheiro-ardida & - & 13,3 & $\mathrm{Q} / \mathrm{T}$ & $\mathrm{H}$ & A \\
\hline Capsicum frutescens $\mathrm{L}$. & Pimenta-malagueta, pimenta-dedo-de-moça & - & 26,6 & $\mathrm{Q} / \mathrm{R}$ & $\mathrm{H}$ & A \\
\hline Capsicum spp. & $\begin{array}{l}\text { Pimenta-malagueta, pimenta-dedo-de-moça, pimenta-de-cheiro, } \\
\text { chumbinho. }\end{array}$ & 13,3 & 6,6 & $\mathrm{Q}$ & $\mathrm{H}$ & A \\
\hline Solanum gilo Raddi & Jiló & 0,74 & 10 & $\mathrm{Q} / \mathrm{T}$ & $\mathrm{H}$ & A \\
\hline Solanum lycopersicum $\mathrm{L}$. & Tomate & 5,9 & 36,6 & $\mathrm{Q}$ & $\mathrm{H}$ & A \\
\hline Solanum melongena $\mathrm{L}$. & Berinjela & 0,74 & - & Q & $\mathrm{H}$ & A \\
\hline Solanum paniculatum L. & Jurubeba & 0,74 & 6,6 & $\mathrm{Q}$ & $\mathrm{AB}$ & $\mathrm{P}$ \\
\hline VITACEAE & & & & & & \\
\hline Vitis sp. & Uva & - & 10 & Q & $\mathrm{L}$ & $\mathrm{P}$ \\
\hline ZINGIBERACEAE & & & & & & \\
\hline Curcuma longa $\mathrm{L}$. & Açafrão & 2,2 & - & Q & $\mathrm{H}$ & A \\
\hline INDETERMINADAS & & & & & & \\
\hline 1 & Fruta-da-Amazônia & - & 3,3 & $\mathrm{Q}$ & - & - \\
\hline 2 & Fruta-banana & 0,74 & - & $\mathrm{Q}$ & - & - \\
\hline 3 & Cacau & 1,5 & - & $\mathrm{Q}$ & - & $\mathrm{P}$ \\
\hline 4 & Coroa-de-frade & - & 3,3 & $\mathrm{Q}$ & A & $\mathrm{P}$ \\
\hline 5 & Gemadinha & 0,74 & - & $\mathrm{Q}$ & - & - \\
\hline 6 & Pêssego & 0,74 & - & $\mathrm{Q}$ & - & - \\
\hline
\end{tabular}

